\title{
Tissue factor expression pattern in human non-small cell lung cancer tissues indicate increased blood thrombogenicity and tumor metastasis
}

\author{
PETRA GOLDIN-LANG $^{1 *}$, QUOC-VIET TRAN $^{1 *}$, IDUNA FICHTNER $^{2}$, ANDREAS EISENREICH $^{1}$, \\ SILVIO ANTONIAK ${ }^{1}$, KARSTEN SCHULZE ${ }^{1}$, SARAH E. COUPLAND ${ }^{3}$, \\ WOLFGANG POLLER $^{1}$, HEINZ-PETER SCHULTHEISS ${ }^{1}$ and URSULA RAUCH ${ }^{1}$

\begin{abstract}
${ }^{1}$ Department of Cardiology and Pneumology, Charité-Universitätsmedizin Berlin, Campus Benjamin Franklin, Berlin; ${ }^{2}$ Max Delbrück Center for Molecular Medicine, Experimental Pharmacology, Berlin-Buch, Germany; ${ }^{3}$ Royal Liverpool Hospital, Department of Cellular and Molecular Pathology, Liverpool, UK
\end{abstract}

Received January 17, 2008; Accepted March 14, 2008

\begin{abstract}
Non-small cell lung cancer (NSCLC) comprises of $75 \%$ of all lung cancers. Human full length tissue factor (flHTF), the physiological initiator of blood coagulation, is aberrantly expressed in certain solid tumors. FIHTF and its soluble isoform, alternatively spliced human tissue factor (asHTF), have been shown to contribute to thrombogenicity of the blood of healthy individuals. The aim of this study was to quantify flHTF and asHTF on mRNA and protein levels (using immunohistochemistry, immunoblotting, and ELISA) on a panel of human NSCLC tissue and plasma specimens. The tissue factor (TF) expression of 21 pulmonary adenomatous (AC) and 12 normal healthy tissues was assessed by real-time qRT-PCR. The TF protein concentration was quantified by ELISA in a subset of $11 \mathrm{AC}$ and 9 normal tissue specimens as well as in the plasma of 13 lung cancer patients and 15 healthy controls. We found a significant increase in the ratio of flHTF/HGAPDH mRNA in AC $(0.24 \pm 0.06$ vs. $0.07 \pm 0.01 ; p=0.02$ vs. controls $)$ and in asHTF/HGAPDH mRNA $(0.027 \pm 0.01$ vs. $0.004 \pm 0.001$; $\mathrm{p}=0.03$ AC vs. controls). AsHTF mRNA expression was significantly lower in patients with stage IA disease compared to patients with higher grade stages, pointing to TF as being a marker of malignancy and metastases. TF protein of lung tumors was significantly increased in $\mathrm{AC}$ ( $\mathrm{p}=0.004$
\end{abstract}

Correspondence to: Dr Petra Goldin-Lang, Charité-Universitätsmedizin Berlin, Charité Centrum 11 für Herz-Kreislauf- und Gefässmedizin, Campus Benjamin Franklin, Medical Clinic II, Department of Cardiology and Pneumology, Hindenburgdamm 30, 12200 Berlin, Germany

E-mail: petra.goldin-lang@charite.de

${ }^{*}$ Contributed equally

Key words: tissue factor, non-small cell lung cancer, thrombogenicity, metastasis vs. controls). TF in plasma was up-regulated in lung cancer patients $(334.9 \pm 95.4$ vs. $124.1 \pm 14.8 \mathrm{pg} / \mathrm{ml}$; $\mathrm{p}=0.02$ vs. controls). Immunohistochemical and immunoblotting data are in line with the increased TF expression, showing elevated blood thrombogenicity of NSCLC patients. The upregulation of flHTF and, especially, asHTF in AC suggests not only a raised risk of thrombosis, but also of tumor progression, thereby, indicating a poor prognosis in these patients.

\section{Introduction}

In the western world, lung cancer is the leading cause of cancer death in men. Lung cancers are classified into several groups, including small-cell lung cancer (SCLC) and nonsmall cell lung cancer (NSCLC). NSCLC comprises of 75\% of all lung cancers. The reason why lung cancer is frequently lethal is that most of the patients are diagnosed in the later stages of the disease, when local infiltrations and metastases are detected and malignancy is incurable. In contrast, outcomes are significantly better if the disease can be detected at an earlier stage, enabling curative resections $(1,2)$.

Tissue factor (TF), a transmembrane glycoprotein comprising of 263 amino acids, plays a key role as a cellular receptor and cofactor of the coagulation factor VII/VIIa, initiating blood coagulation $(3,4)$. TF is not only present in the adventitia of the vessel wall but also in circulating blood, thereby increasing blood thrombogenicity. In vitro studies on the expression of thrombogenic TF showed a secretion of TF-positive microparticles from smooth muscle cells, endothelial cells and monocytes in response to several inflammatory stimuli (3-8). Furthermore, a human alternatively spliced TF isoform (asHTF) in addition to the full length TF (flHTF) was detected in lung tissue. This isoform lacks exon 5, which encodes the transmembrane TF domain. AsHTF is a soluble form of the TF protein and circulates in blood, possibly contributing to blood thrombogenicity as well as angiogenesis (9). Up-regulated levels of circulating TF antigen were found in patients with solid 
tumors and disseminated vascular disease (DIC) $(10,11)$. Upregulated TF levels in the plasma of tumor patients, especially in carcinomas of the pancreas, mamma and lung were associated with an increased rate of thrombotic events $(10,12)$. Moreover, experimental studies showed TF expressing tumor tissues to reveal a more aggressive metastasis formation (13). It has been shown that TF expression in NSCLC cells is associated with vascular endothelial growth factor (VEGF) expression and increased microvessel density. The survival time for patients with immunologically TF-negative NSCLC tumors is longer than with TF-positive tumors (13).

The aim of this study was to quantify the TF isoform expression on mRNA and protein levels with a particular focus on the soluble TF isoform asHTF in adenocarcinoma of the lung. Furthermore, the present study was performed in order to examine the value of the TF isoform expression as a marker of lung cancer stage and progression on a panel of human lung adenocarcinomas and corresponding normal, healthy tissues as well as the plasma of lung cancer patients and healthy controls.

\section{Materials and methods}

Patients and tumors. Twenty-one patients with adenocarcinomas of the lung and 12 normal healthy control tissues of the lung underwent surgery at the Charité-Universitätsmedizin Berlin, Campus Benjamin Franklin. Each patient gave written informed consent. The histological classification of the tumors was based on the World Health Organization Study (14) and was carried out by two pathologists. Each tumor was staged at the time of their surgery according to the guidelines of the International Union Against Cancer (15).

Clinical information was obtained from a review of hospital charts or from the respective hospital tumor registry. The study was approved by the Ethics Committee of the Charité University Hospital.

TF isoform-specific real-time PCR (Taq Man). Total RNA of human NSCLC tissue specimens was isolated by the TRIzol method according to manufacturer's instructions (Invitrogen, Karlsruhe, Germany), reverse transcribed including a control without Reverse Transcriptase (First Strand cDNA synthesis kit for RT-PCR [AMV]) to exclude a generation of genomic DNA and analyzed by PCR using primers as previously described (16).

Western blot. Samples from human lung AC tissues were homogenized in a radio-immunoprecipitation assay buffer, supplemented with $1 \%$ protease inhibitor cocktail (P-8340, Sigma Aldrich, Munich, Germany) and were separated by electrophoresis on a $12 \%$ sodium dodecyl sulfate-polyacrylamide gel (SDS-PAGE). The gels were transferred to a PVDF membrane (Immobilon-P, Millipore GmbH, Schwalbach, Germany), which was blocked in 5\% nonfat dry milk diluted in tris-buffered saline. Blots were incubated at $4^{\circ} \mathrm{C}$ overnight with the primary polyclonal anti-TF antibody (pAb-sTF, $6 \mu \mathrm{g} / \mathrm{ml}$ ) (16). Washed blots were incubated for 90 min with a secondary horseradish peroxidase-conjugated antibody (DakoCytomation, Hamburg, Germany). Washed blots were subjected to the ECL Plus Western blotting detection reagent (Amersham, Freiburg, Germany) for detection of the immunoreactive signal by chemiluminescence and the membranes were then exposed to X-ray film (Kodak X-OMAT AR, Kodak, Stuttgart, Germany). The membranes were stripped in $100 \mathrm{mM}$ glycine $(\mathrm{pH} 2.9)$ at room temperature for $45 \mathrm{~min}$. The stripped blots were blocked again for $1 \mathrm{~h}$ and incubated with a primary polyclonal antibody anti-asHTF (dilution at 1:100, Pineda AntikörperService) (16). After incubation, the blots were washed and treated as described. The membrane was then exposed to Xray film.

Protein extraction and TF ELISA. Human lung cancer tissue samples were homogenized in a glass potter containing $100 \mu \mathrm{l}$ ice-cold radio-immunoprecipitation assay buffer, supplemented with $1 \%$ protease-inhibitor cocktail, incubated on ice for $30 \mathrm{~min}$ and centrifuged at $10,000 \mathrm{x}$ g for $10 \mathrm{~min}$ at $4^{\circ} \mathrm{C}$. Total protein content of the cell lysate was measured using a bicinchoninic acid protein assay according to the manufacturer's protocol (Pierce, Rockford, IL). The protein was precipitated with acetone and afterwards re-suspended in TBS (pH 8.5). To quantify the total TF protein content (flHTF and asHTF) in the tumor specimens, TF ELISA was performed according to the instruction manual (Imubind tissue factor ELISA kit, American Diagnostica Inc.). Separate plasma aliquots obtained from lung cancer patients and healthy subjects were quickly thawed and utilized for the Imubind tissue factor ELISA kit (American Diagnostica Inc., Stamford, CT).

Immunohistochemistry. A total of 12 patients with adenocarcinomatous lung cancer who underwent lung resection were studied. Histological diagnoses were made on the basis of sections stained with haematoxylin and eosin. The TNM classification is used internationally for malignant tumors and is accepted by the American Joint Committee on Cancer (AJCC) and the Union Internationale Contre le Cancer (UICC) $(17,18)$. The specimens were compared with negative controls. The results were graded as follows: negative $(0)$, moderately positive (1), positive (2) and strongly positive (3).

For flHTF and asHTF isoform staining, specimens were immediately snap-frozen and stored at $-80^{\circ} \mathrm{C}$. Cryostat sections were washed with phosphate-buffered saline (PBS), incubated in $4 \% \mathrm{H}_{2} \mathrm{O}_{2}$ and immunostained either with polyclonal rabbit anti-human antibody against sTF (American Diagnostica Inc.), diluted at 1:75 in PBS/fetal calf serum, or with polyclonal rabbit anti-human antibody against asHTF (diluted at 1:50), respectively. A second polyclonal antibody specific for asHTF as previously described (16) was used for staining. After incubation with the primary antibody, specimens were washed, incubated with an appropriate biotinylated secondary antibody (DakoCytomation) and counterstained with haematoxylin (Merck, Darmstadt, Germany). For detection, the Vectastain ABC kit was used according to the manufacturer's instructions (Vector Laboratories, Burlingame, CA).

Statistical analysis. Data analysis was performed using GraphPad Prism 4.0 for Windows. To test data distribution 
A

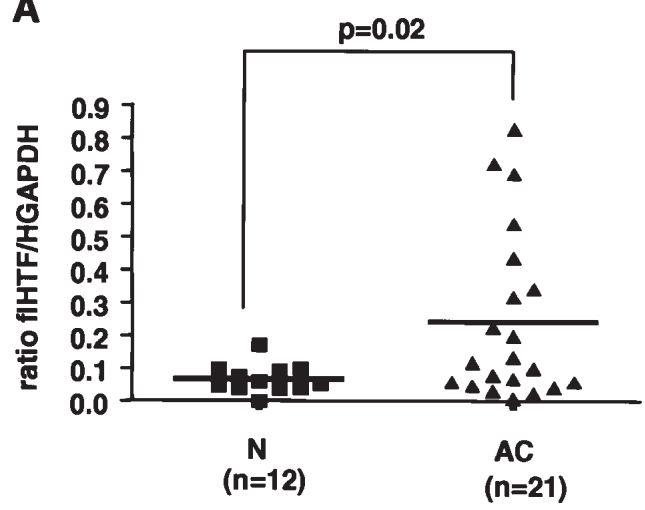

B

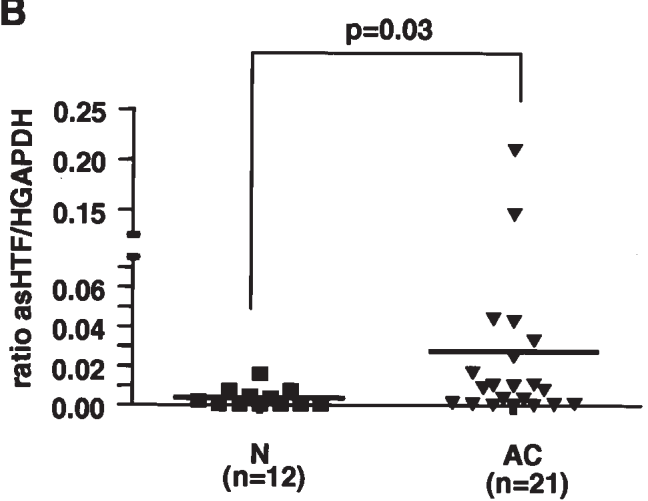

Figure 1. mRNA expression of flHTF (A) and asHTF (B) of AC vs. normal healthy (N) lung control tissues. (A) N vs. AC: p=0.02 (n=12-21). (B) N vs. AC: $\mathrm{p}=0.03(\mathrm{n}=12-21)$.

the Kolmogorov-Smirnov test was performed. Values are represented as mean \pm standard error of mean (SEM) or median and interquartile range for non-parametric data. For parametric data, the statistical significance of differences between the groups was determined by applying an unpaired Student's t-test. The Mann-Whitney U test was performed for non-parametric data. The Chi-square test evaluated the association between the low TF group and the high TF group with tumor characteristics. We determined the cut-off for high and low TF groups on the basis of flHTF and asHTF mRNA expression. $\mathrm{P} \leq 0.05$ was considered significant.

\section{Results}

Elevated TF mRNA expression in lung adenocarcinomas. A total of 21 human adenocarcinomas (AC) and 12 normal human tissues were examined for mRNA expression of flHTF, asHTF and human glyceraldehyde-3-phosphate dehydrogenase (HGAPDH) genes using quantitative real-time RT-PCR (qRT-PCR). A 3.4-fold increase of mRNA expression was yielded in $\mathrm{AC}$ vs. control specimens (ratio flHTF/ HGAPDH $0.24 \pm 0.06$ vs. $0.07 \pm 0.01 ; \mathrm{p}=0.02$ vs. controls) (Fig. $1 \mathrm{~A}$ ). The ratio asHTF/HGAPDH in AC $(0.027 \pm 0.01$ vs. $0.004 \pm 0.001$; $\mathrm{p}=0.03$ vs. controls) was 6.8 -fold raised compared with the controls (Fig. 1B).

Patient characteristics of flHTF and asHTF mRNA expression groups. A total of 21 patients were divided into two groups with a cut-off value of flHTF/HGAPDH ratio of 0.08 and asHTF/HGAPDH ratio of 0.008 . The flHTF group and asHTF groups, respectively, were determined according to the mRNA expression analysis by real-time qRT-PCR. Mean age of the lung cancer population at the time of analysis was $65.6 \pm 0.6$ years (range $41-82$ years). Fourteen patients were male and 7 were female. A majority of AC patients in the high flHTF group (58.3\%) and $54.5 \%$ of the high asHTF group patients had stage II to IV cancers. Ten out of 21 (47.6\%) had lymph node metastases. Nine out of 21 (42.9\%) patients with AC revealed recurrent disease after surgery (Table I). FlHTF mRNA expression was not significantly altered between the flHTF groups (Table IA). In contrast, asHTF mRNA expression was significantly lower in patients with stage IA disease compared to patients with higher grade stages ( $\mathrm{p} \leq 0.01$ vs. stage IIIA, IIIB) (Table IB).

Immunohistochemistry of adenocarcinomas of the lung. Snap-frozen lung cancer tissues of 12 patients were examined by immunohistochemistry for human flHTF and asHTF. Eight out of $12 \mathrm{AC}(66.7 \%)$ were moderately positive for flHTF protein expression. One of the $12 \mathrm{AC}$ revealed a strongly positive asHTF expression whereas throughout a moderately positive staining in 11 out of 12 tumors (91.7\%) with the asHTF-specific antibody was observed vs. the overall negative control healthy tissue.

Western blot analysis of lung cancer tumor specimens with TF full length- and TF isoform-specific antibodies. FlHTF and asHTF were detected by polyclonal anti-TF antibodies (pAb-sTF, $6 \mu \mathrm{g} / \mathrm{ml}$ ) directed against the extracellular domain of the TF protein (Fig. 3) yielding a single protein band at $46 \mathrm{kDa}$ (Fig. 3A, lanes 1 and 2) for flHTF and at $28 \mathrm{kDa}$ for asHTF (Fig. 3B, lanes 1 and 2). For equal loading of the protein, HGAPDH expression was shown in Fig. 3C. FlHTF protein expression in $\mathrm{AC}$ compared to the healthy control was increased. AsHTF was detected in AC, though not in normal, healthy controls.

Up-regulation of TF protein concentration in lung adenocarcinoma specimens and corresponding healthy control tissues. A significant increase in cellular TF protein measured by TF ELISA became prominent in AC $(n=11)$ with a TF level of $1119.0 \pm 210.3 \mathrm{pg} / \mathrm{ml}(\mathrm{p}=0.004)$ compared to healthy control tissue specimens $(n=9)$ with a TF level of $324.0 \pm 92.5 \mathrm{pg} / \mathrm{ml}$ (Fig. 2B). TF protein expression in the plasma of NSCLC tumor patients $(n=13)$ was demonstrated to be significantly up-regulated with $\mathrm{TF}$ protein levels of $334.9 \pm 95.44$ vs. $124.1 \pm 14.79 \mathrm{pg} / \mathrm{ml}(\mathrm{p}=0.02)$ in the healthy controls $(n=15)$.

\section{Discussion}

In the present study, we demonstrated flHTF and especially asHTF mRNA expression and TF protein levels in lung AC tissues compared to healthy tissues to be significantly elevated. 
A

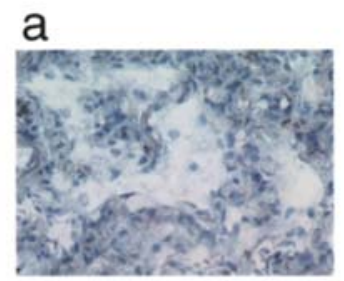

b

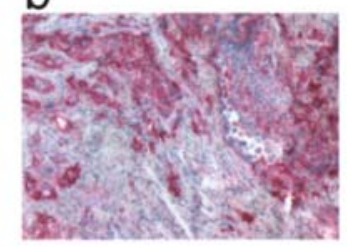

C

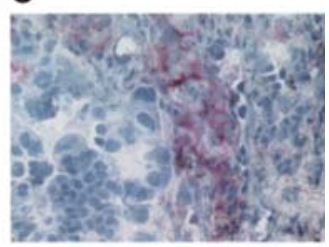

B

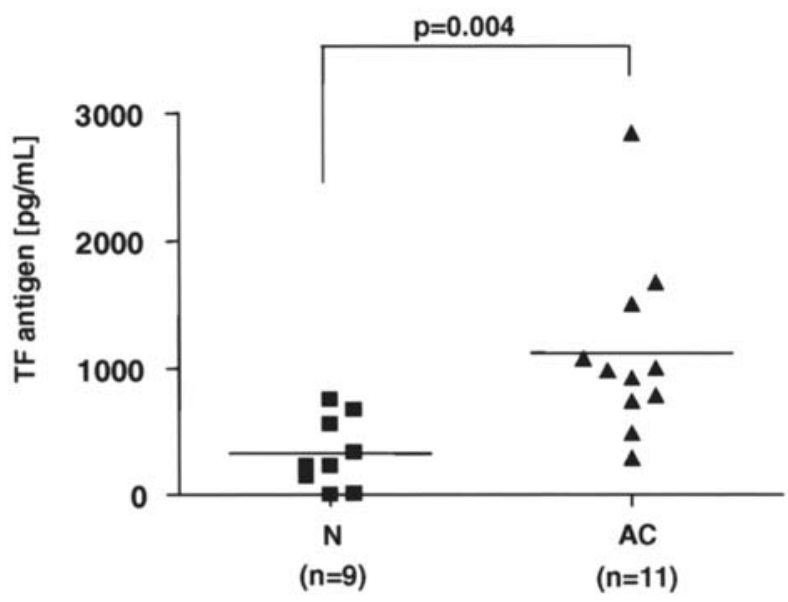

Figure 2. (A) Cryopreserved sections of human adenocarcinomas (AC) of the lung. (a) Normal healthy human lung tissue (magnification, x400). (b) AC stained with psTF Ab (magnification, x200). (c) AC stained with asTF Ab (magnification, x400). (B) Intratumoral concentrations of total TF antigen in normal control tissues $(\mathrm{N})$ and adenocarcinomas of the lung (AC). $\mathrm{N}$ vs. AC: $\mathrm{p}=0.004(\mathrm{n}=9-11)$.

1

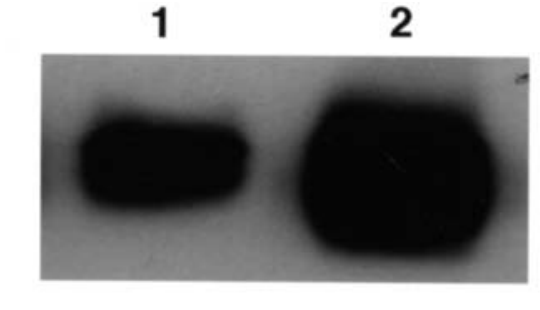

\section{A fIHTF}

\section{B asHTF}

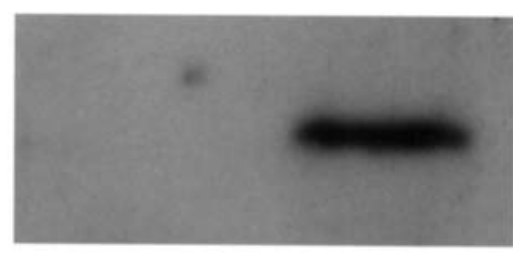

\section{HGAPDH}

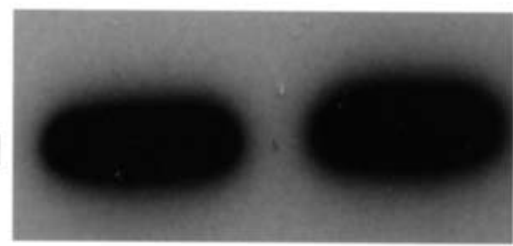

N

AC

Figure 3. Representative Western blot analysis of human NSCLC tumor specimens. (A) flHTF protein expression of human AC tumor specimens detected with the polyclonal anti-TF antibody (pAb-sTF), lane 1, healthy control; lane 2, AC. (B) AsHTF protein expression of human AC tumor specimens detected with the polyclonal anti-asHTF antibody, lane 1, healthy control; lane 2, AC. (C) Human GAPDH protein expression as loading control of equal protein amounts, lane 1 , healthy control; lane 2, AC.
Furthermore, in addition to the significant increase in intratumoral TF protein, plasma TF levels of patients with $\mathrm{AC}$ increased when compared to the plasma of the healthy controls.

Immunostaining for TF revealed lung carcinomas, such as bronchoalveolar cell carcinomas and small-cell lung cancer to express TF irrespective of their location (17). Neoplastic cells invading the basement membrane or completely obliterating the alveolar architecture stained at the same intensity as those confined to the bronchial mucosa (17). Volm et al (18) reported the expression of the TF protein to be significantly higher in AC compared to SCC of NSCLC. Sawada et al (19) found human NSCLC cell lines derived from metastatic cells producing high levels of TF in vitro. Furthermore, they studied surgically-resected specimens and found significantly stronger staining for TF in NSCLC specimens with metastases than in those without. Koomagi and Volm (13), in contrast, did not find any correlation to metastasis in lung cancer patients. A significant association between TF expression and high microvessel density was observed in several tumor types including lung cancers. Moreover, patients with TF-positive lung carcinomas were found to have shorter survival times than patients with TF-negative carcinomas (13). The studies with NSCLC tumors are based on immunohistochemistry and describe the TF protein expression without distinguishing between the two TF isoforms, flHTF and asHTF.

To date, little is known about the impact of asHTF in human lung cancer. AsHTF is a protein soluble in aequeous solutions, though the functional activity and the biological relevance still remain unclear. In our study, the TF levels in the 
Table I. Patient characteristics.

\begin{tabular}{cc}
\hline A, Low and high flHTF groups & \\
\hline Low flHTF group & High flHTF group \\
\hline flHTF/HGAPDH & flHTF/HGAPDH \\
$\leq 0.08(\mathrm{n}=9)$ & $>0.08(\mathrm{n}=12)$
\end{tabular}

Total: $\mathrm{n}=21$

\section{Age (years) \\ $<66$ \\ $\geq 66$}

Gender

Male

Female

Histopathology

$\mathrm{AC}$

Stage

IA

IB

IIA

IIB

IIIA

IIIB

IV

Lymph node metastases

$$
\begin{aligned}
& \mathrm{LN}(-) \\
& \mathrm{LN}(+) \text { (N 1-3) }
\end{aligned}
$$

Recurrence after surgery

\begin{tabular}{lll} 
No & 2 & 0 \\
Yes & 3 & 6 \\
n.e. & 4 & 6 \\
\hline
\end{tabular}

tumor itself were at least 3-fold higher than in the normal controls. It is known that levels of TF plasma are elevated in cancer patients and experimental studies have shown that TF may contribute to thrombus formation $(3,20)$. TF plasma concentrations in patients with $\mathrm{AC}$ of the lung were found to be on average 2.7-fold that of normal controls. TF in blood may derive from asHTF or from flHTF-containing microparticles (MPs), which is secreted by activated cells, thereby contributing to systemic thrombogenicity. It has been reported that MPs bearing active TF are released readily from tumor cells and can significantly activate coagulation even at low concentrations (21). Recently, we showed that human asHTF is expressed and the expression is up-regulated in squamous cell carcinoma of the lung (22). Beuneu et al (23) reported asHTF mRNA to be expressed in a human adenocarcinomatous pancreatic duct cell line. They hypothesized that procoagulant activity measured in the supernatant of duct cells was ascribed to asHTF as well as TF-bearing microparticles (MPs) (23). In vitro experiments on colorectal and squamous cell carcinoma revealed the shedding of TF-positive MPs rather than soluble TF isoform, asHTF, to
Table I. continued.

B, Low and high asHTF groups.

\begin{tabular}{cc}
\hline Low asHTF group & High asHTF group \\
\hline asHTF/HGAPDH & asHTF/HGAPDH \\
$\leq 0.008(\mathrm{n}=10)$ & $>0.008(\mathrm{n}=11)$ \\
\hline
\end{tabular}

Total: $\mathrm{n}=21$

$\begin{array}{lll}\text { Age (years) } & 3 & \\ <66 & 7 & 7 \\ \geq 66 & & \\ \text { Gender } & 8 & 6 \\ \quad \text { Male } & 2 & 5 \\ \text { Female } & & \\ \text { Histopathology } & 10 & 11 \\ \text { AC } & & \\ \text { Stage } & 5^{\text {a }} \\ \text { IA } & 1 & 0 \\ \text { IB } & 0 & 5 \\ \text { IIA } & 2 & 0 \\ \text { IIB } & 0 & 1 \\ \text { IIIA } & 0 & 3 \\ \text { IIIB } & 2 & 2 \\ \text { IV } & & 0 \\ \text { Lymph node metastases } & 6 & 5 \\ \text { LN (-) } & & \\ \text { LN (+) (N 1-3) } & 4 & 6 \\ \text { Recurrence after surgery } & & \\ \text { No } & 0 & 5 \\ \text { Yes } & 5 & 5 \\ \text { n.e. } & 5 & \\ \end{array}$

AC, Adenocarcinoma of the lung; LN (-), lymph node negative; LN (+), lymph node positive; n.e., not evaluable; ${ }^{\mathrm{a}} \mathrm{p} \leq 0.01$ (vs. IIIA, IIIB).

be the main source of TF released from human cancer cells (12). In addition to the two known TF isoforms, a novel human TF splice variant has recently been described to be present in tumor cells. Approximately $10 \%$ of the total TF transcripts in the tumor consisted of this novel TF variant (TF-A) formed by the insertion of a $495 \mathrm{bp}$ sequence, referred to as exon IA, which is derived from an internal sequence of the first intron (24). However, the novel TF mRNA transcript TF-A was found to be differentially expressed in various tumor cells. Hence, its suitability as a diagnostic marker for solid tumors has been considered, though has not yet been verified.

Recent studies have shown that asHTF-positive tumors seem to have increased vascular density compared with controls, suggesting a role of asHTF as promoting angiogenesis. AsHTF was postulated to be the more important TF isoform in the enhancement of tumor growth (25) TF, though not flHTF, enhances tumor growth and tumor-associated vasculature (25). In line with our data, asHTF expression was only seen in patients with an AC tumor of a higher advanced 
stage than IA. TF expression in cancer tissue has been suggested to enhance the transcription of VEGF, which stimulates angiogenesis thus playing a role in the cellular signalling involved in the tumor growth and metastatic potential of certain cancers (26). To gain a deeper knowledge of the mechanisms and pathways, the role of TF as a general marker of activated endothelium in pathological angiogenesis and the phosphorylation state of cellular TF, in particular in invasive areas of the carcinoma, should be further examined (27).

In conclusion, this study demonstrated that flHTF and, at least to the same degree, asHTF may serve as a risk factor in lung cancer patients. Further investigation with a larger sample size will be needed to evaluate whether specific TF isoforms in tumors and tumor-associated blood vessels may present targets for anti-cancer drug design.

\section{Acknowledgements}

We would like to thank Miriam Boots for her support in the tumor specimen collection and documentation and Franziska Bleis for her technical assistance. This study was supported by grants: Sonderforschungsbereich Transregio 19 'Inflammatorische Kardiomyopathie-Molekulare Pathogenese und Therapie' Teilprojekt A3 (U. Rauch, S. Antoniak).

\section{References}

1. Alberg AJ, Brock MV and Samet JM: Epidemiology of lung cancer: looking to the future. J Clin Oncol 23: 3175-3185, 2005.

2. Hoffman PC, Mauer AM and Vokes EE: Lung cancer. Lancet 355: 479-485, 2000.

3. Giesen PL, Rauch U, Bohrmann B, et al: Blood-borne tissue factor: another view of thrombosis. Proc Natl Acad Sci USA 96: 2311-2315, 1999.

4. Rauch U, Bonderman D, Bohrmann B, et al: Transfer of tissue factor from leukocytes to platelets is mediated by CD15 and tissue factor. Blood 96: 170-175, 2000.

5. Rauch U and Nemerson Y: Tissue factor, the blood, and the arterial wall. Trends Cardiovasc Med 10: 139-143, 2000.

6. Rauch U, Osende JI, Fuster V, Badimon JJ, Fayad Z and Chesebro JH: Thrombus formation on atherosclerotic plaques: pathogenesis and clinical consequences. Ann Intern Med 134: 224-238, 2001.

7. Rauch U and Nemerson Y: Circulating tissue factor and thrombosis. Curr Opin Hematol 7: 273-277, 2000.

8. Mallat Z, Hugel B, Ohan J, Leseche G, Freyssinet JM and Tedgui A: Shed membrane microparticles with procoagulant potential in human atherosclerotic plaques: a role for apoptosis in plaque thrombogenicity. Circulation 99: 348-353, 1999.

9. Bogdanov VY, Balasubramanian V, Hathcock J, Vele O, Lieb M and Nemerson Y: Alternatively spliced human tissue factor: a circulating, soluble, thrombogenic protein. Nat Med 9: 458-462, 2003.
10. Osterud B and Bjorklid E: The tissue factor pathway in disseminated intravascular coagulation. Semin Thromb Hemost 27: 605-617, 2001.

11. Lip GY, Chin BS and Blann AD: Cancer and the prothrombotic state. Lancet Oncol 3: 27-34, 2002.

12. Yu JL and Rak JW: Shedding of tissue factor (TF)-containing microparticles rather than alternatively spliced TF is the main source of TF activity released from human cancer cells. J Thromb Haemost 2: 2065-2067, 2004.

13. Koomagi R and Volm M: Tissue-factor expression in human non-small-cell lung carcinoma measured by immunohistochemistry: correlation between tissue factor and angiogenesis. Int J Cancer 79: 19-22, 1998.

14. Greene FL, Page DL, Fleming ID, Fritz AG, Balch CM, Haller DG, et al: AJCC Cancer Staging manual (eds). 6th edition Springer-Verlag, New York, pp167-177, 2002.

15. Sobin LH and Wittekind CH: International Union Against Cancer. TNM classification of malignant tumors (eds). 6th edition Wiley-Liss, New York, 2002.

16. Szotowski B, Goldin-Lang P, Antoniak S, Bogdanov VY, Pathirana D, Pauschinger M, Dorner A, Kuehl U, Coupland S, Nemerson Y, Hummel M, Poller W, Hetzer R, Schultheiss HP and Rauch U: Alterations in myocardial tissue factor expression and cellular localization in dilated cardiomyopathy. J Am Coll Cardiol 45: 1081-1089, 2005.

17. Callander NS, Varki N and Rhao LVM: Immunohistochemical identification of tissue factor in solid tumors. Cancer 70 : 1194-1201, 1992.

18. Volm M, Koomagi R, Efferth T and Mattern J: Protein expression profiles of non-small cell lung carcinomas: correlation with histological subtype. Anticancer Res 22: 2321-2324, 2002.

19. Sawada M, Miyake S, Ohdama S, et al: Expression of tissue factor in non-small-cell lung cancers and its relationship to metastasis. Br J Cancer 79: 472-477, 1999.

20. Kakkar AK, DeRuvo N, Chinswangwatanakul V, Tebbutt S and Williamson RC: Extrinsic-pathway activation in cancer with high factor VIIa and tissue factor. Lancet 346: 1004-1005, 1995.

21. Tesselaar ME, Romijn FP, Van der Linden IK, Prins FA, Bertina RM and Osanto S: Microparticle-associated tissue factor activity: a link between cancer and thrombosis? J Thromb Haemost 5: 520-527, 2007.

22. Rauch U, Antoniak S, Boots M, Schulze K, Goldin-Lang P, Stein H, Schultheiss HP and Coupland SE: Association of tissue-factor upregulation in squamous-cell carcinoma of the lung with increased tissue factor in circulating blood. Lancet Oncol 6: $254,2005$.

23. Beuneu C, Vosters O, Movahedi B, et al: Human pancreatic duct cells exert tissue factor-dependent procoagulant activity: relevance to islet transplantation. Diabetes 53: 1407-1411, 2004.

24. Chand HS, Ness SA and Kisiel W: Identification of a novel human tissue factor splice variant that is upregulated in tumor cells. Int J Cancer 118: 1713-1720, 2006.

25. Hobbs JE, Zakarija A, Cundiff DL, et al: Alternatively spliced human tissue factor promotes tumor growth and angiogenesis in a pancreatic cancer tumor model. Thromb Res 120 (Suppl 2): S13$21,2007$.

26. Zhang Y, Deng Y, Luther T, Müller M, Ziegler R, Waldherr R, Stern DM and Nawroth PP: Tissue factor controls the balance of angiogenic and antiangiogenic properties of tumor cells in mice. J Clin Invest 94: 1320-1327, 1994.

27. Belting M, Ahamed J and Ruf W: Signaling of the tissue factor coagulation pathway in angiogenesis and cancer. Arterioscler Thromb Vasc Biol 25: 1545-1550, 2005. 